\title{
Shuttle mechanism for charge transfer in Coulomb blockade nanostructures
}

\author{
L. Y. Gorelik,,${ }^{1,2}$ A. Isacsson, ${ }^{1}$ M. V. Voinova, ${ }^{1,3}$ B. Kasemo, ${ }^{1}$ R. I. Shekhter,${ }^{1}$ and M. Jonson ${ }^{1}$ \\ (1) Department of Applied Physics, Chalmers University of Technology and Göteborg University, S-412 96 Göteborg, Sweden \\ (2) B. Verkin Institute for Low Temperature Physics and Engineering, 310164 Kharkov, Ukraine \\ (3) Kharkov State University, 310077 Kharkov, Ukraine
}

Room-temperature Coulomb blockade of charge transport through composite nanostructures containing organic interlinks has recently been observed. A pronounced charging effect in combination with the softness of the molecular links implies that charge transfer gives rise to a significant deformation of these structures. For a simple model system containing one nanoscale metallic cluster connected by molecular links to two bulk metallic electrodes we show that self-excitation of periodic cluster oscillations in conjunction with sequential processes of cluster charging and decharging appears for a sufficiently large bias voltage. This new 'electron shuttle' mechanism of discrete charge transfer gives rise to a current through the nanostructure, which is proportional to the cluster vibration frequency.

PACS numbers: 73.23.Hk, 72.80.Tm, 72.80.Le

The term Coulomb blockade refers to a supression of the tunneling current through metallic grains embedded in a dielectric matrix. The origin of this phenomenon lies in the fundamental quantization of charge in units of $e$ and it occurs in systems with small quantum charge fluctuations. As a result the redistribution of grain charges necessarily associated with a current flow can only be made in quantized units of $e$. The correspondingly quantized electrostatic charging energy, which for small nanoscale grains can be large compared to other relevant energies related to temperature and bias voltage, tends to block the current. A number of different Coulomb blockade-based phenomena have been discovered - typically at very low temperatures - over the last decade or so [1,2], largely due to the developments within nanotechnology.

In this Letter we discuss a new type of composite mesoscopic structure demonstrating Coulomb blockade behavior at room temperatures 33 . 5. The crucial aspect of these structures from the point of view of our work is that they contain metallic grains or molecular clusters with a typical size of $1-5 \mathrm{~nm}$ that can vibrate; their positions are not necessarily fixed. This is because the dielectric material surrounding them is elastic and consists of mechanically soft organic molecules. These molecular inter-links have elastic moduli which are typically two or three orders of magnitude smaller than those of ordinary solids $\sqrt{6}$. Their ohmic resistance $R$ is high and of order $10^{7}-10^{8} \mathrm{ohm}$, while at the same time they are extremely small - a few nanometers in size.

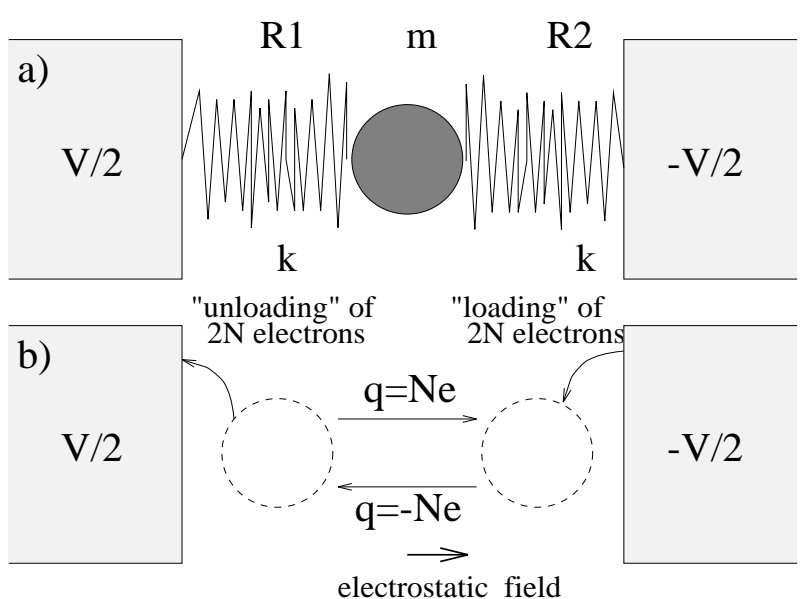

FIG. 1. (a) Simple model of a soft Coulomb blockade system in which a metallic grain (center) is linked to two electrodes by elastically deformable organic molecular links. (b) Dynamic instabilities occur since in the presence of a sufficiently large bias voltage $V$ the grain is accelerated by the same electrostatic force towards first one, then the other electrode. A cyclic change in direction is caused by the repeated "loading" of electrons near the negatively biased electrode and the subsequent "unloading" of the same at the positively biased electrode. As a result the sign of the net grain charge alternates leading to an oscillatory grain motion and a novel "electron shuttle" mechanism for charge transport.

A large Coulomb blockade effect in combination with the softness of the dielectric medium implies that charge transfer may give rise to a significant deformation of these structures as they respond to the electric field associated with a bias voltage. We have found that self-excitation of mechanical grain vibrations accompanied by barrier deformations is possible for a sufficiently large bias voltage. As we show below this gives rise to a novel 'shuttle mechanism' for electron transport. The point is that a grain (the shuttle) oscillates between two turning points. One of them is located near a positively- and the other near a negatively biased electrode. Due to the Coulomb blockade phenomenon an integer number of electrons are loaded onto the grain close to one turning point and the same number of electrons are unloaded close to the other as illustrated in Fig. 1. The result is that in each cycle the shuttle moves a discrete number of electrons from one electrode to the other. It follows that the current is proportional to the mechanical vibration frequency of the grain.

One can estimate the typical frequency $\omega$ of such vi- 
brations using elastic moduli for the relevant organic molecules. For a metallic cluster a few nanometers in diameter and a molecular length of $1 \mathrm{~nm}$ the characteristic vibration frequency is of the order of $10^{10}-10^{11} \mathrm{~s}^{-1}$. Hence it can be of the order of or higher than the typical charge fluctuation frequency $\nu=1 / R C$ (here $C$ is the capacitance of the metallic cluster which for room temperature Coulomb blockade systems is of the order $\left.10^{-18}-10^{-19} \mathrm{~F}\right)$. This means that charge fluctuations and cluster vibrations become strongly coupled in such systems.

For illustration we consider the simplest possible interesting system. It consists of one small metallic grain connected by elastic molecular links to two bulk leads on either side, as shown in Fig. 1a. The tunnel junctions between the leads and the grain are modeled by tunneling resistances $R_{1}(x), R_{2}(x)$ which are assumed to be exponential functions of the grain coordinate $x$. In order to avoid unimportant technical complications we study the symmetric case for which $R_{1,2}=R e^{ \pm x / \lambda}$. When the position of the grain is fixed, the electrical potential of the grain and its charge $q$ are given by current balance between the grain and the leads [1]. As a consequence, at a given bias voltage $V$ the charge $q$ is completely controlled by the ratio $R_{1}(x) / R_{2}(x)$. In addition the bias voltage generates an electrostatic field $\mathcal{E}=\alpha V$ in the space between the leads [7] and hence a charged grain will be subjected to an electrostatic force $F_{q}=\alpha V q$.

The central point of our considerations is that the grain - because of the "softness" of the organic molecular links connecting it to the leads - may move and change its position. The grain motion disturbs the current balance and as a result the grain charge will vary in time. This variation affects the work $W=\alpha V \int \dot{x} q(t) d t$ performed on the grain during, say, one period of its oscillatory motion. It is significant that this work is nonzero and positive, i.e., the electrostatic force, on the average, accelerates the grain. The nature of this acceleration is best understood by considering a grain oscillating with a large amplitude $A>\lambda$. In this case the charge fluctuations between the grain and the most distant electrode is exponentially suppressed when the displacement of the grain is maximal. As a result the grain at such a turning point gains extra charge from the nearby electrode, as shown in Fig. $1 \mathrm{~b}$. The added charge is positive at the turning point near the positively charged electrode and negative at the turning point close to the negatively charged one. The sign change of the charge takes place mainly in the immediate vicinity of the electrodes while along the major part of the path between them the charge on the grain is fixed and determined by the most recently encountered electrode. Therefore the grain acts as a shuttle that carries positive charge on its way from the positive to the negative electrode and negative charge on its return trip. The electrostatic force is hence at all times directed along the line of motion and thus accelerates the grain. This state- ment is true also for small amplitude oscillations. The result is an electro-mechanical instability in the sense that even if the grain is initially at rest a sufficiently strong electrostatic field will cause the grain to start vibrating.

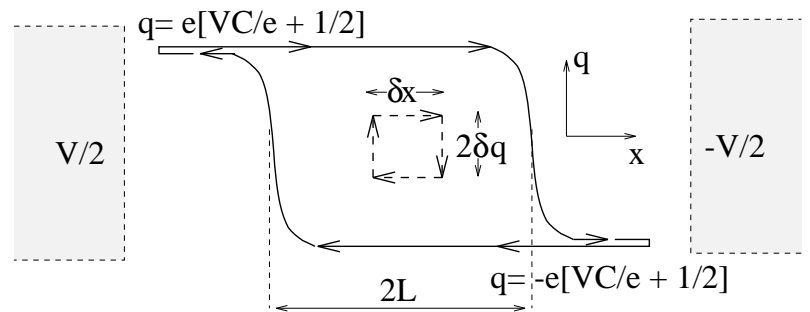

FIG. 2. Charge response to a cyclic grain motion. The dashed arrows describe an imagined, particularily simple trajectory in the charge-position plane that allows the work done on the grain by the electrostatic field to be easily calculated and shown to be positive, hence leading to an instability: For times $t<-\tau$ when the grain is at rest at $x=-\delta x / 2$ the charge exchange with the positively biased electrode dominates and the grain is positively charged $q=\delta q$; at the instant $t=-\tau$ it instantaneously moves to the point $x=+\delta x / 2$ where the charge relaxes to a new equlibrium value $q=-\delta q$; then the negatively charged grain instantaneously moves back at $t=+\tau$. During this cyclic process the electrostatic force acts only along the direction of the grain displacement. The solid line describes (realistically) the charge response at large oscillation amplitudes. At $|x|>L$ the charge exchange with the far lead is exponentially suppressed and the charge on the grain takes the fixed quantized value corresponding to thermal equilibrium with the nearest lead. As a result no net energy is pumped into the system.

To be more precise one can show that for small deviations from equilibrium $(x=0, q=0)$ and provided $q(t)$ is defined as the linear response to the grain displacement, $q(t)=\int \chi\left(t-t^{\prime}\right) x\left(t^{\prime}\right) d t^{\prime}$, the work done on the grain is positive. To prove this one may consider the simple closed trajectory in $x, q$-space shown with dashed arrows in Fig. 2. For this trajectory it follows trivially that the work $W$ is positive. Since this result can be shown [8] to be independent of the particular trajectory considered, this simplifying choice does not imply any restriction on its validity. The positive value of $W$ at small vibration amplitudes implies that self-excitation of mechanical vibrations will occur when a finite voltage bias $V$ is applied to our system.

In real systems a certain amount $Q$ of energy is dissipated due to mechanical damping forces which always exist. In order to get to the self-excitation regime more energy must be pumped into the system from the electrostatic field than can be dissipated; $W$ must exceed $Q$. Since the electrostatic force increases with the bias voltage this condition can be fullfiled if $V$ exceeds some critical value $V_{c}$. If the electrostatic and damp- 
ing forces are much smaller than the elastic restoring force self-excitation of vibrations with a frequency equal to the eigenfrequency of elastic oscillations arise. In this case $V_{c}$ can be implicitly defined by the relation $\omega \gamma=\alpha V_{c} \operatorname{Im} \chi(\omega)$,

where $\omega \gamma$ is the imaginary part of the complex dynamic modulus. In the general case, when the charge response is determined by Coulomb-blockade phenomena, $\chi$ is an increasing but rather complicated function of $V$ and there is no way to solve for $V_{c}$ analytically. However, one can establish the value of $V_{c}$ with an uncertainty given by the Coulomb blockade voltage $V_{e}=e / C$ :

Above the threshold voltage the oscillation amplitude will increase exponentially until a balance between dissipated and absorbed energy is achieved and the system reaches a stable self-oscillating regime [9]. The amplitude $A$ of the self-oscillations will therefore be determined by the criterion $W(A)=Q(A)$. Let us now consider large amplitude oscillations. Then, due to the exponentially strong dependence of the tunnel resistances on the grain position, no net energy is absorbed by the system outside the region $|x|<L=\ln A \omega / \lambda \nu<A$ (see Fig. 2). Therefore, the amount of absorbed energy in this limit does not depend strongly on the amplitude $A$ [10]. This results in a saturation of the energy that can be pumped into the system, which is of the order $W \sim 4 \alpha V q_{e q} L$. Here $q_{e q}$ is the magnitude of the charge that obtains if the grain is in thermal equilibrium with one lead. In the Coulomb blockade regime, at temperatures $k_{B} T \ll e^{2} / C$, the value of $q_{e q}$ is quantized in units of $e: q_{e q}=N e=[V C / e+1 / 2] e$. To estimate the dissipated energy we take $Q(A)=\pi \omega \gamma A^{2}$, which corresponds to the simple phenomenological damping force $F_{d}=-\gamma \dot{x}$. This approach leads to the following expression for the amplitude of self-oscillation when $V \gg V_{c}$ : $A \sim \lambda \Omega V C / e(\gamma \omega)^{1 / 2}$ ( $\Omega$ is defined in Fig. 3). One can see from this expression that the self-oscillation grows in amplitude with increasing bias voltage.

In the fully developed self-oscillating regime the oscillating grain, sequentially moving electrons from one lead to the other, provides a new 'shuttle mechanism' for charge as shown in Fig. 1b. In each cycle $2 N$ electrons are transferred, so the average contribution to the current from this shuttle mechanism is

$$
I=2 e N f, \quad N=\left[\frac{C V}{e}+\frac{1}{2}\right],
$$

where $f \equiv \omega / 2 \pi$ is the self-oscillation frequency. This current does not depend on the tunneling rate $\nu$. The reason is that when the charge jumps to or from a lead, the grain is so close that the tunneling rate is large compared to the elastic vibration frequency. Hence the shuttle frequency - not the tunneling rate - provides the 'bottle neck' for this process.

We emphasize that the current due to this shuttle mechanism can be substantially larger than the conven- tional current via a fixed grain. This is the case when $\omega \gg \nu$. Our analysis shows that the electromechanical instability discussed above has dramatic consequences for the current-voltage characteristics of single electron transistor configurations as shown in Fig. 3. Even for a symmetric double junction, where no Coulomb staircase appears in conventional designs, we predict that the shuttle mechanism for charge transport manifests itself as a current jump at $V=V_{c}$ and as a Coulomb staircase as the voltage is further increased.

To support the qualitative arguments given above we have performed analytical and numerical analyses based on the simultaneous solution of Newton's equation,

$$
m \ddot{x}=-k x-\gamma \dot{x}+\alpha V q, q=e \sum_{n} n P_{n},
$$

for the motion of a grain of mass $m$ and a Master equation for the charge distribution function $P_{n} \equiv \operatorname{Tr}\{\delta(n-\hat{n}) \hat{\rho}\}$ ( $\hat{n}$ is the electron number operator and $\hat{\rho}$ is the density matrix of electrons),

$$
\begin{aligned}
& \frac{2}{\nu} \dot{P}_{n}=e^{-x / \lambda} \Gamma(n-1, n) P_{n-1}+e^{x / \lambda} \Gamma(n+1, n) P_{n+1} \\
& \quad-e^{-x / \lambda} \Gamma(n, n+1) P_{n}-e^{x / \lambda} \Gamma(n, n-1) P_{n}
\end{aligned}
$$

The quantities $P_{n}$ completely describe the "charge" state of the grain in the Coulomb blockade regime and hence the average current through the system

$$
I=\frac{\nu}{2 T} \int_{0}^{T} e^{-x / \lambda} \sum_{n} \Gamma(n, n+1) P_{n} d t
$$

The dimensionless tunneling rates $\Gamma(n \mp 1, n)$ in Eq. (3) are given by

$$
\Gamma(n \mp 1, n)=\left(\frac{C V}{e} \mp n+\frac{1}{2}\right) \theta\left(\frac{C V}{e} \mp n+\frac{1}{2}\right) .
$$

and numerically obtained $I-V$ curves for different amounts of damping are shown in Fig 3.

A more precise (and time consuming) calculation along the line $l$ sketched in Fig. 3 is shown in Fig. 4. Rather than solving the Master Eq. (3) we here calculate the current utilizing a Monte Carlo type algorithm. The nonmonotonic behaviour of the current along this line is due to competition between the two charge transfer mechanisms present in the system, the ordinary tunnel current and the mechanically mediated current $I_{\text {mech }}\left(x_{0}, t\right)=$ $\delta\left(x(t)-x_{0}\right) \dot{x}(t) q(t)$ through some cross section at $x_{0}$. We define the shuttle current as the time average mechanical current through the plane located at $x_{0}=0$. This current together with the tunnel current for the same cross section is shown in Fig. 4. As the damping in the system is reduced the oscillation amplitude grows and the shuttle current is enhanced while the ordinary tunneling current is suppressed. In the limit of low damping this leads to a quantization of the total current in terms of $2 e f$. 


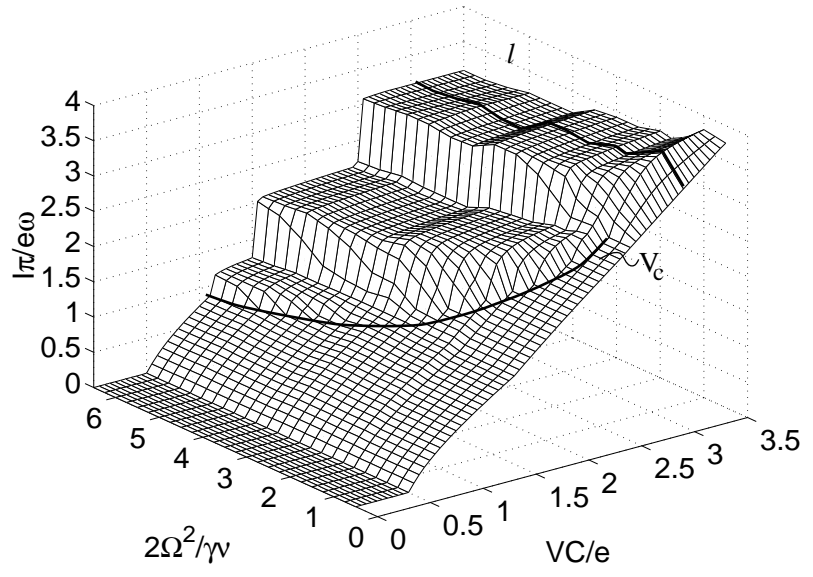

FIG. 3. Current due to the proposed shuttle mechanism through the composite Coulomb blockade system of Fig. 1. The current is normalized to the eigenfrequency $\omega$ of elastic grain vibrations and plotted as a function of normalized bias voltage $V$ and inverse damping rate $\gamma^{-1}\left(\Omega=\sqrt{\alpha e^{2} / \lambda C}\right)$. With infinite damping no grain oscillations occur and no Coulomb staircase can be seen. The critical voltage $V_{c}$ required for the grain to start vibrating is indicated by a line.

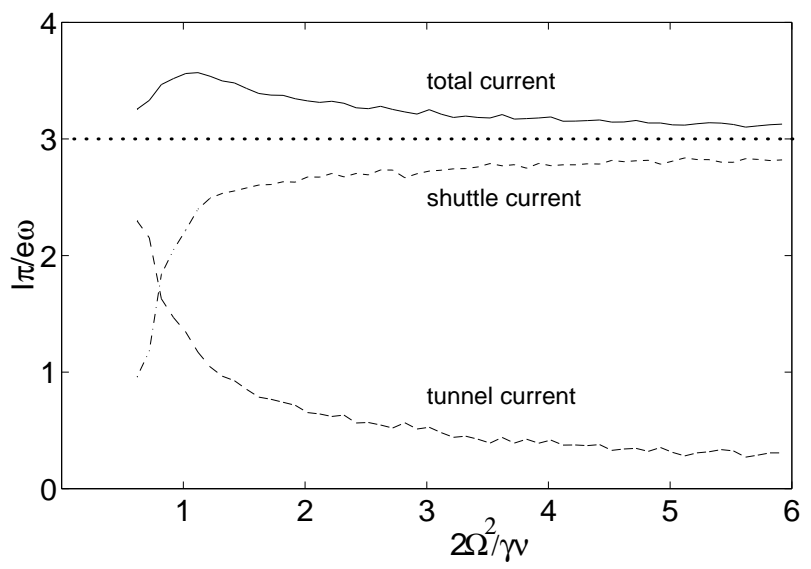

FIG. 4. Cross section along the line $l$ in Fig. 3. The total time averaged current consist of two parts, the shuttle current and the tunneling current. The time averaged shuttle current is the mechanically transfered current through the center of the system $\langle\delta(x(t)) \dot{x}(t) q(t)>$, the remaining part comes from ordinary tunneling. As the inverse damping $\gamma^{-1}$ increase the shuttle current approaches the quantized value $I \pi / e \omega=3$. The tunnel current is proportional to the fraction of the oscillation period spent in the middle region, $|x|<\lambda$. This fraction is inversely proportional to the oscillation amplitude and hence the tunnel current decreases as $\gamma^{-1}$ increases. The fine structure in the results is due to numerical noise.

In conclusion we have performed a numerical analysis of the charge transport by the shuttle mechanism through the soft Coulomb blockade system shown in Fig. 1. The following results summarize the discussion above: (i) a dynamical instability exists above a critical bias voltage $V_{c}$; (ii) a limit cycle in the position-charge plane exists for the grain (shuttle) motion above $V_{c}$ leading to a novel 'electron shuttle' mechanism for charge transport; (iii) The current-voltage curve has a step-like structure, a type of Coulomb staircase, even for a symmetric system. Our numerical analysis was made for a symmetric case for which $R_{1}(0)=R_{2}(0)$. However all phenomena discussed above — with small quantitative corrections - still appear in the asymmetric case when $R_{1}(0) \neq R_{2}(0)$, which is of great importance for experiments. In a strongly asymmetric situation when $R_{1}(0) \gg R_{2}(0)$ the electromechanical instability still exist but instead of one threshold voltage $V_{c}$ a set of instability "windows" associated with the $I-V$ steps 11] will appear. This work has been supported by the Swedish TFR, KVA, and NFR.

[1] I. O. Kulik and R. I. Shekhter, Sov. Phys. JETP 41, 308 (1975).

[2] D. V. Averin and K. K. Likharev, in Mesoscopic Phenomena in Solids, edited by B. L. Altshuler, P. A. Lee, and R. A. Webb (Elsevier, Amsterdam, 1991), p.173.

[3] R. P. Andres, T. Bein, M. Dorogi, S. Feng, J. I. Henderson, C. P. Kubiak, W. Mahoney, R. G. Osifchin, and R. Reifenberger, Science 272, 1323 (1996).

[4] D. L. Klein, J. E. B. Katari, R. Roth, A. P. Alivisatos, and P. L. McEuen Appl. Phys. Lett. 68, 2574 (1996).

[5] E. S. Soldatov, V. V. Khanin, A. S. Trifonov, S. P. Gubin, V. V. Kolesov, D. E. Presnov, S. A. Iakovenko, and G. B. Khomutov, JETP Lett. 64, 556 (1996).

[6] O. Alvarez, R. Lattore Biophys.J. 21, 1, (1978); V. I. Pasechnic, T. Gyanik, Sov. Biophyzika 22, 941 (1977).

[7] In reality the capacitances of the system and hence its electrostatic energy $E(x)$ depend on the grain position. By expanding $E(x)$ for fixed $q$ and $V$ and retaining only the linear term one gets the position independent effective electrostatic field used here.

[8] According to general properties of linear response (see for example L. D. Landau and E. M. Lifshitz Statistical Physics (Pergamon, Oxford, 1980) sign $\{\omega \operatorname{Im} \chi(\omega)\}=$ $\operatorname{sign}\left\{\int d \omega\left|x_{\omega}\right|^{2} \omega \operatorname{Im} \chi(\omega)\right\}=$ Const. From this it immediately follows that the sign of $W$ does not depend on the type of cyclic motion actually realized and that it can be established by considering the simplest possible path.

[9] Our numerical simulation show that instability develops into a self-oscillatory regime corresponding to a limiting cycle rather than to a more complicated attractor or even chaotic motion.

[10] A more sophisticated treatment shows that $W \propto \ln A$ at large amplitude.

[11] J. Wan, K. McGreer, L. I. Glazman, A. Goldman, and R. I. Shekhter, Phys. Rev. B 43, 9381 (1991). 